\title{
A Gas-forming Liver Abscess with Massive Bleeding into the Abscess Cavity Due to a Ruptured Inferior Phrenic Artery
}

\author{
Mari Satoh, Takayuki Kogure, Akinobu Koiwai, Daisuke Fukushi, Morihisa Hirota, \\ Katsuya Endo and Kennichi Satoh
}

\begin{abstract}
:
An 88-year-old woman developed a huge abscess, forming an air-fluid level in the right lobe of the liver. A pigtail catheter was placed and drained thick pus with putrid odor from the abscess cavity. Gram-positive rods were detected in the pus, which were subsequently determined to be Clostridium perfringens by culture. She developed hemorrhaging in the abscess cavity when the right inferior phrenic artery was damaged by inflammation that had spread from the abscess. Emergency transarterial embolization with gelatin sponges was performed, and the bleeding ceased. We herein report a rare case of liver abscess that caused inferior phrenic artery injury, resulting in bleeding.
\end{abstract}

Key words: liver abscess, Clostridium perfringens, massive arterial bleeding, inferior phrenic artery

(Intern Med 60: 3913-3919, 2021)

(DOI: 10.2169/internalmedicine.7746-21)

\section{Introduction}

Pyogenic liver abscesses are associated with gas formation in $5.6 \%$ to $31.8 \%$ of cases (1). Approximately $68 \%$ of gas-forming liver abscesses are caused by Klebsiella pneumoniae, followed by Escherichia coli, with a very low incidence of induction by Clostridium perfringens (2). C. perfringens infection causes hemolysis, leading to a critical condition $(3,4)$. Thus, its early diagnosis is essential to avoid severe complications.

The diagnosis of $C$. perfringens as the cause of infection is challenging because these bacteria are natural inhabitants of the intestine and vagina in humans. One uncommon complication of liver abscess is bleeding of the hepatic arteries due to pseudoaneurysm (5). Inflammation of the abscess spreads and damages arteries, leading to bleeding. However, there have been no reports of bleeding from arteries other than those in the liver in association with liver abscess.

We herein report a case of gas-forming liver abscess caused by $C$. perfringens, resulting in bleeding into the abscess cavity due to right inferior phrenic arterial injury.

\section{Case Report}

An 88-year-old woman was referred to our division with a large cystic lesion in the right lobe of the liver. Abdominal computed tomography (CT) showed a massive abscess 15.5 $\mathrm{cm}$ in size that contained an air-fluid level in the right lobe of the liver. She had a medical history of total hip replacement surgery at 76 years old. She was on medication for type 2 diabetes, hypertension, hyperlipidemia, and hypothyroidism. She had slight epigastric discomfort at the referral but did not show any other symptoms, such as abdominal pain, nausea, or diarrhea.

At a physical examination, she showed conjunctival icterus, but her body temperature, blood pressure, pulse rate, and respiratory rate were intact. The laboratory tests suggested intense inflammation with increased white blood cell (WBC) count and C-reactive protein (CRP). She also showed severe liver injury with elevated levels of bilirubin and liver enzymes (Table). She had normocytic anemia with a hemoglobin level of $8.0 \mathrm{~g} / \mathrm{dL}$ and a mean corpuscular volume of $96 \mathrm{fL}$. We considered the anemia to be secondary to 
Table. Clinical Characteristics on Admission.

\begin{tabular}{|c|c|}
\hline White blood cells $(/ \mu \mathrm{L})$ & 50,600 \\
\hline Red blood cells $\left(\times 10^{4} / \mu \mathrm{L}\right)$ & 281.0 \\
\hline Hemoglobin (g/dL) & 8.0 \\
\hline Hematocrit $(\%)$ & 25.5 \\
\hline MCV (fL) & 90.8 \\
\hline $\mathrm{MCH}(\mathrm{pg})$ & 28.4 \\
\hline MCHC (\%) & 31.3 \\
\hline Platelet $\left(\times 10^{4} / \mu \mathrm{L}\right)$ & 25.0 \\
\hline Prothrombin time (\%), INR & $52.4 \%, 1.52$ \\
\hline APTT (s) & 38.6 \\
\hline $\mathrm{FDP}(\mu \mathrm{g} / \mathrm{mL})$ & 14.2 \\
\hline Antithrombin-III (\%) & 67.0 \\
\hline Total bilirubin (mg/dL) & 4.7 \\
\hline Direct bilirubin $(\mathrm{mg} / \mathrm{dL})$ & 3.7 \\
\hline AST (IU/L) & 1,209 \\
\hline ALT (IU/L) & 696 \\
\hline LDH (IU/L) & 639 \\
\hline ALP (IU/L) & 803 \\
\hline$\gamma$-GTP (IU/L) & 41 \\
\hline Total protein $(\mathrm{g} / \mathrm{dL})$ & 4.9 \\
\hline Albumin $(\mathrm{g} / \mathrm{dL})$ & 1.5 \\
\hline Glucose (mg/dL) & 99 \\
\hline Hemoglobin A1c (\%) & 6.2 \\
\hline Urea nitrogen $(\mathrm{mg} / \mathrm{dL})$ & 34.0 \\
\hline Creatinine (mg/dL) & 1.08 \\
\hline Sodium $(\mathrm{mEq} / \mathrm{L})$ & 133 \\
\hline Potassium $(\mathrm{mEq} / \mathrm{L})$ & 5.2 \\
\hline Chloride (mEq/L) & 104 \\
\hline C-reactive protein $(\mathrm{mg} / \mathrm{dL})$ & 23.6 \\
\hline Procalcitonin (ng/mL) & 5.71 \\
\hline
\end{tabular}

MCV: mean corpuscular volume, $\mathrm{MCH}$ : mean corpuscular hemoglobin, MCHC: mean corpuscular hemoglobin concentration, APTT: activated partial thromboplastin time, FDP: fibrin-fibrinogen degradation products, AST: aspartate aminotransferase, ALT: alanine aminotransferase, LDH: lactate dehydrogenase, ALP: alkaline phosphatase, $\gamma$-GTP: $\gamma$-glutamyl transpeptidase

persistent infection and liver injury. Her elevated bilirubin level was predominantly direct bilirubin; however, her aspartate aminotransferase (AST) level was extremely high compared to the levels of alanine aminotransferase (ALT), and her lactate dehydrogenase (LDH) and serum potassium levels were also increased. Although hemolytic anemia could not be ruled out, we presumed that the disease was not severe enough to induce a critical condition.

Abdominal CT showed a large abscess $15.5 \mathrm{~cm}$ in diameter that contained gas and fluid forming an air-fluid level in the right lobe of the liver (Fig. 1). We detected no bile duct abnormalities as the possible cause of jaundice. Ultrasoundguided percutaneous drainage of the abscess was performed on admission, wherein a 7.0-French pigtail catheter was inserted into the abscess cavity through a right intercostal space (Fig. 2A). A total of $120 \mathrm{~mL}$ of thick, brown, purulent fluid with a putrid odor was drained from the abscess cavity. Intravenous administration of biapenem, a broad-spectrum antibiotic, was started immediately. A microscopic examination of the pus with Gram staining detected large Grampositive, rod-shaped bacteria (Fig. 2B).

Given the gas formation and Gram-positive rods, we suspected Clostridia infection as the cause of the liver abscess and started the intravenous administration of Clindamycin and Metronidazole in combination. A bacterial culture of the pus subsequently identified $C$. perfringens. We diagnosed the patient with a gas-forming massive liver abscess caused by $C$. perfringens. Her jaundice and ALT elevation suggested severe organ damage caused by $C$. perfringens infection. The pus was highly viscous and drained very slowly through the pigtail catheter. We injected 20 to $40 \mathrm{~mL}$ of saline through the catheter twice a day.

Fourteen days after the drainage catheter placement and intravenous administration of antibiotics, the patient's WBC count returned to the normal level, and her CRP level markedly decreased. Her jaundice was resolved, and the ALT level decreased to within the normal limit (Fig. 3); however, the cavity of the abscess remained $13.6 \mathrm{~cm}$ in diameter, filled with a fluid-like component and the gas forming the air-fluid level.

Twenty-six days after the drainage catheter placement, she developed a sudden loss of consciousness. Although head CT revealed no abnormalities, abdominal CT detected hemorrhaging in the cavity coming from a branch of the right inferior phrenic artery. Inflammation spreading from the abscess cavity to the diaphragm might have damaged the artery (Fig. 4). The drainage catheter was located at the dorsal side of the abscess cavity some distance from the bleeding site (Fig. 4), so vascular damage due to contact with a longterm-placed drainage tube seemed unlikely.

Emergency angiography detected contrast extravasation from the right inferior phrenic artery (Fig. 5). A branch of the right inferior phrenic artery showed oozing hemorrhaging and a round, enhanced lesion, suggesting a pseudoaneurysm (Fig. 5B, C). A microcatheter was placed in the right inferior phrenic artery, and spherical gelatin sponges $1 \mathrm{~mm}$ in diameter were administered until the flow of contrast agent into the abscess cavity disappeared. After successful embolization, the patient's state of consciousness stabilized without rebleeding.

We continued administering antibiotics for another 16 days until the fluid stopped draining from the catheter tube. Follow-up CT showed that the abscess cavity was still present in the liver, with a size of $11.7 \mathrm{~cm}$, but the gas that had been forming the air-fluid level had disappeared (Fig. 6). The abscess cavity was still occupied by a substance similar to pus and containing small, low-density areas of air. We suspected the substance with small air bubbles in the abscess cavity to be solidified purulent fluid. The infection seemed to have been resolved, as the WBC count and CRP level had almost normalized, so we removed the drainage 

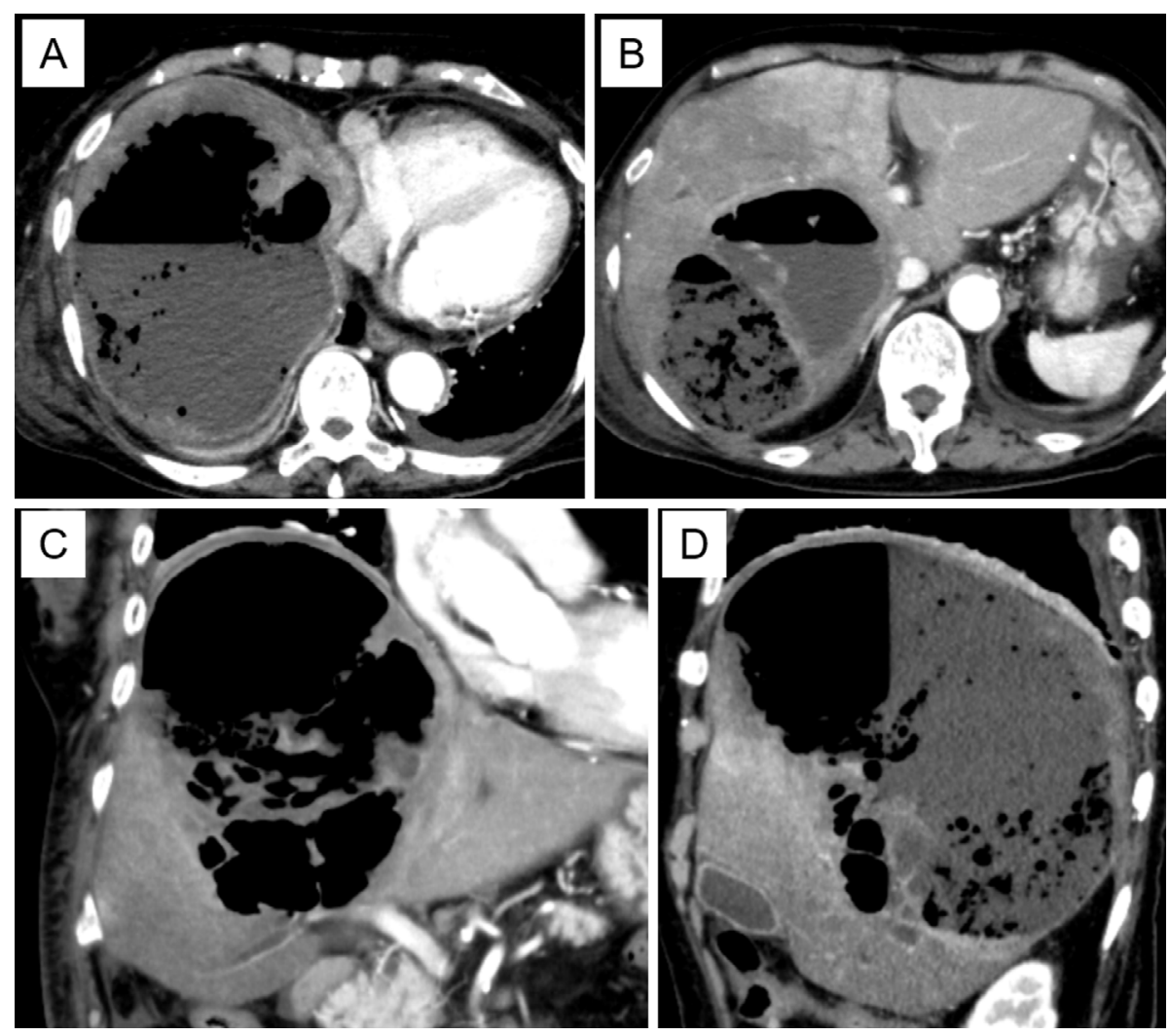

Figure 1. Abdominal CT images at referral to our division. A large abscess $15.5 \mathrm{~cm}$ in size containing gas and fluid, forming an air-fluid level, was located in the right lobe of the liver. (A) Axial image, cephalic level. (B) Axial image, caudal level. (C) Coronal image. (D) Sagittal image.
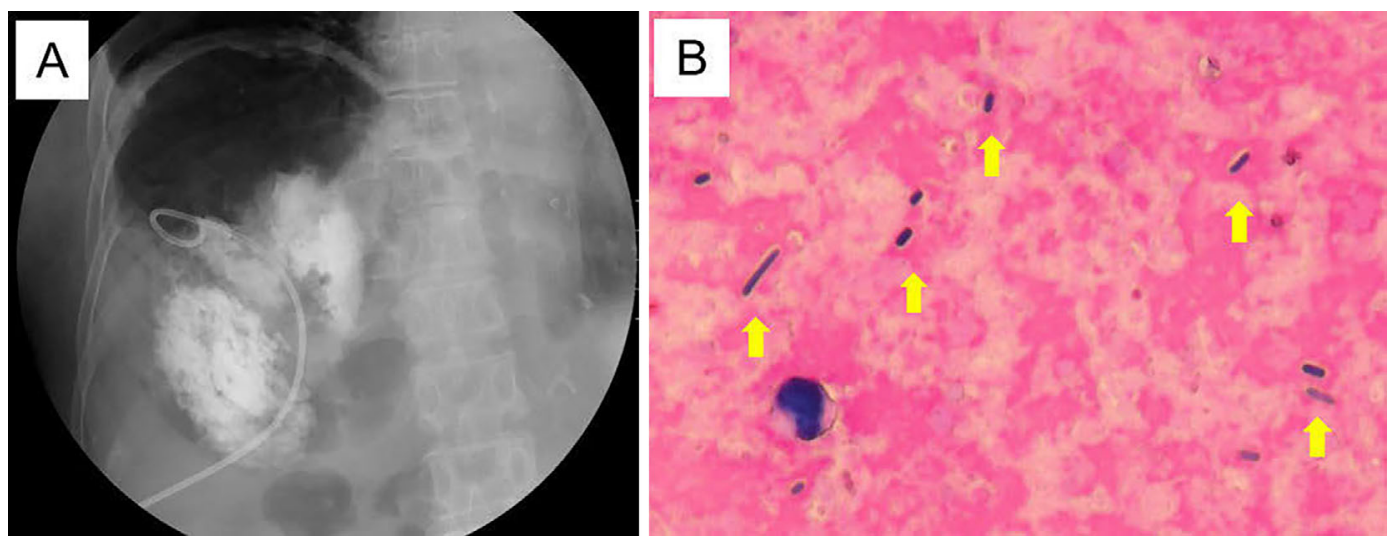

Figure 2. Fluoroscopic image of the abscess cavity and microscopic images of the pus with Gram staining. (A) Fluoroscopic image of the abscess with pigtail drainage catheter and injected contrast media. (B) Microscopic image of the pus with Gram staining. Large, Gram-positive, rod-shaped bacteria were detected in the pus (arrows).

catheter tube. The intravenous antibiotic was changed to oral levofloxacin for two weeks.

The patient remained stable after discontinuing oral levofloxacin without a relapse of inflammation (Fig. 3). She was discharged from our hospital 12 days after oral levofloxacin withdrawal and transferred to the referral hospital for rehabilitation. We confirmed that her CRP level had returned to normal 26 days after she moved to the referral hospital.

\section{Discussion}

C. perfringens is a normal enteric and vaginal inhabitant in humans and animals and is widely distributed in soil as well as fresh and salt water. C. perfringens is a Grampositive, anaerobic, rod-shaped bacteria that causes gas gangrene. It produces alpha-toxin and perfringolysin $\mathrm{O}$, which are believed to induce the development of gas gangrene in soft tissues (6). In addition to gas gangrene, the most com- 


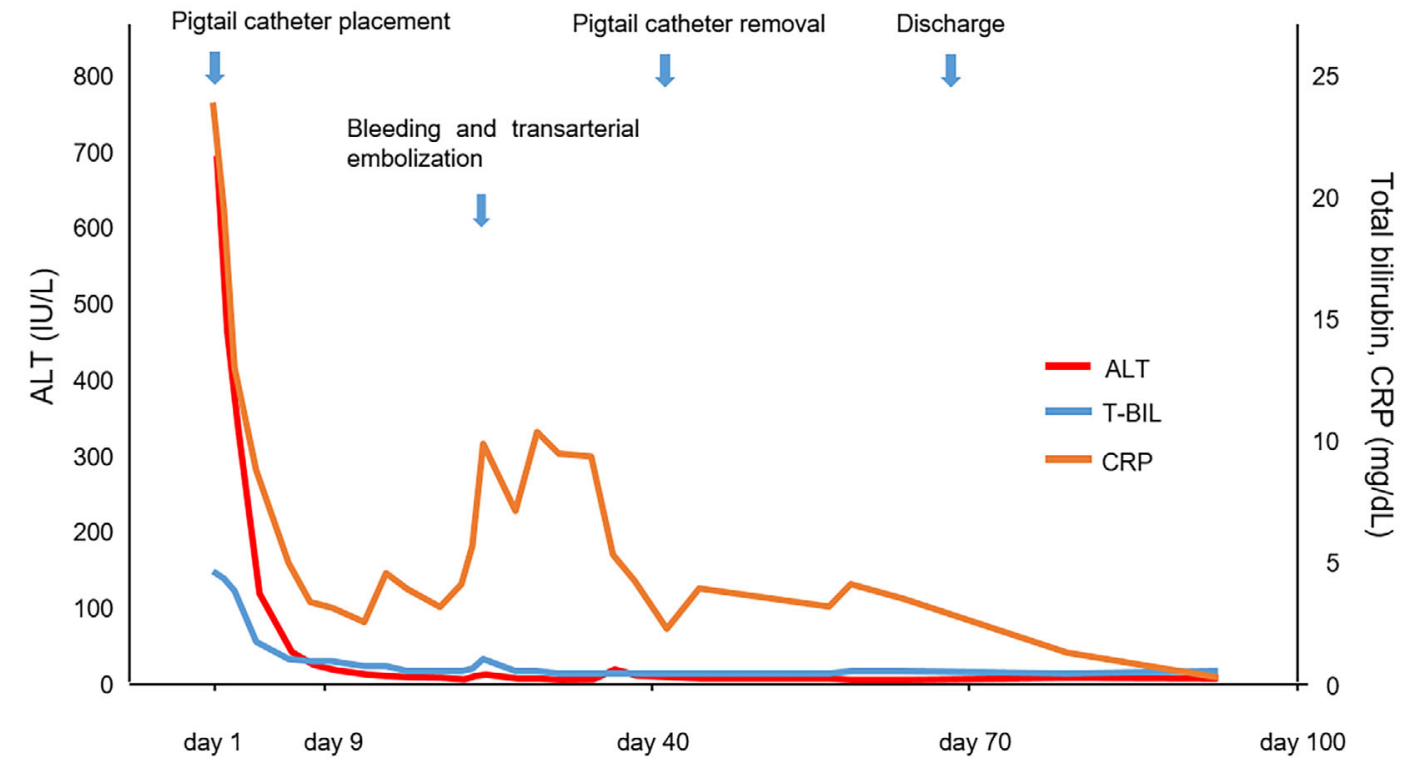

Figure 3. Clinical course. ALT: alanine aminotransferase, T-BIL: total bilirubin, CRP: C-reactive protein, BIPM: biapenem, MTZ: metronidazole, CLDM: clindamycin, CMZ: cefmetazole, SBT/ ABPC: sulbactam/ampicillin, LVFX: levofloxacin

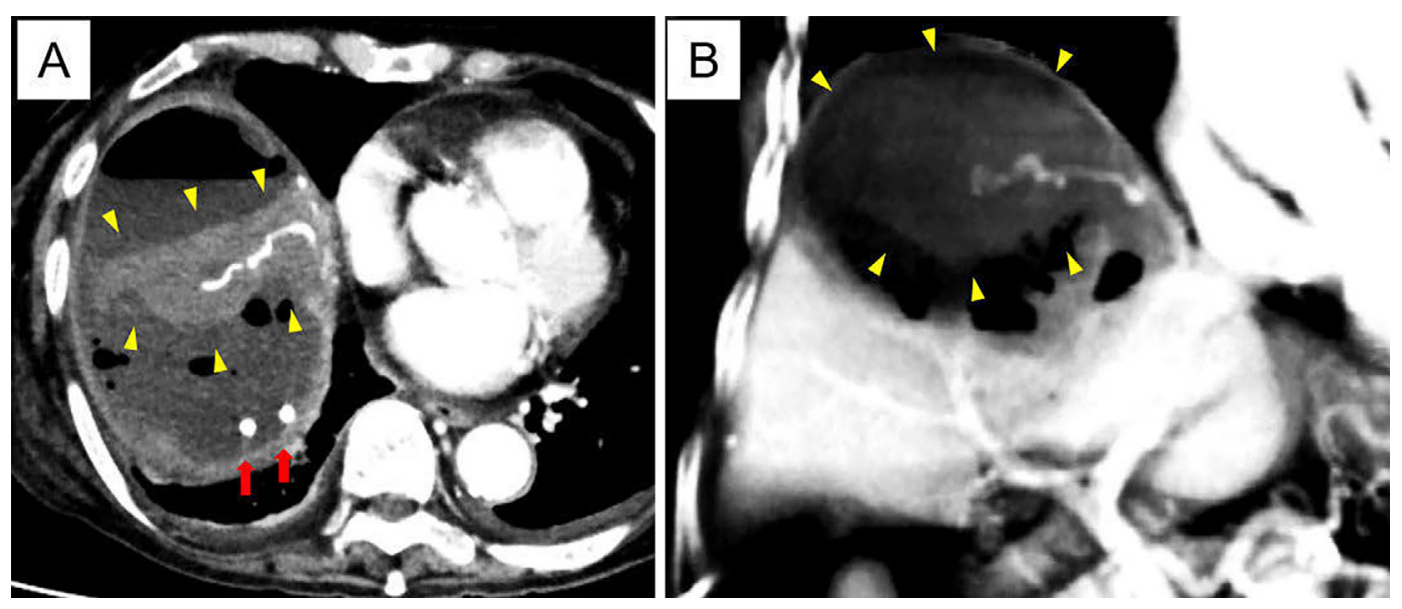

Figure 4. Abdominal CT images during hemorrhaging. Mild hyperdensity substances indicating bleeding from the right inferior phrenic artery were detected in the abscess cavity (arrowheads). Cross-sections of the pigtailed drainage catheter can be seen (red arrows). (A) Axial image. (B) Coronal image.

mon disease caused by $C$. perfringens is foodborne disease, and liver abscess is extremely rare (7). K. pneumoniae and E. coli are the leading causes of gas-forming liver abscess, with Clostridium species a rare cause (8). The most common infecting route of liver abscess is through the bile ducts and portal vein. Since $C$. perfringens is a normal inhabitant in the gastrointestinal tract, the infection in the present case appears to have occurred via the biliary tract or portal vein. The present patient did not have any risk factors for bacterial translocation, such as biliary disease, enterocolitis, or colon cancer. She was an older compromised host with pre- existing diseases, such as type 2 diabetes, which may have caused her to develop the liver abscess with $C$. perfringens.

Liver abscesses caused by $C$. perfringens has an extremely poor prognosis $(9,10)$. It can induce jaundice due to severe hepatocyte injury and is often fatal, causing acute massive intravascular hemolysis (11-13). Kwon et al. reported that hemolysis occurs in $70 \%$ of liver abscesses caused by $C$. perfringens, and the mortality rate is $68 \%$ (14). Although the prompt initiation of treatment is crucial, an early diagnosis is challenging because bacterial culture identification of $C$. perfringens usually takes several 

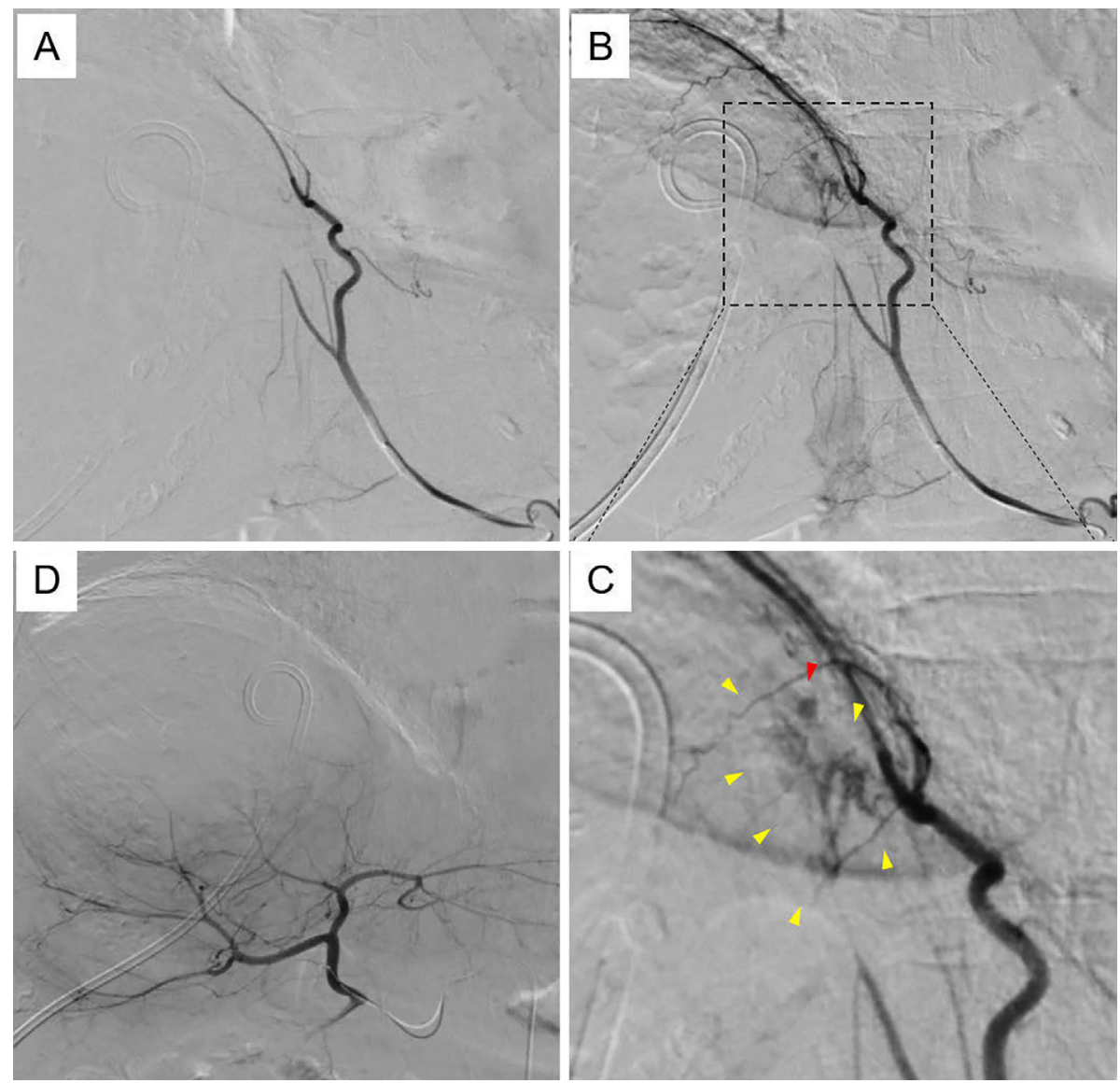

Figure 5. Digital subtraction angiography. (A) Right inferior phrenic artery (RIPA) angiogram, early phase. (B, C) RIPA angiogram in the late phase showing contrast extravasation. A branch of the right inferior phrenic artery showed oozing hemorrhaging (yellow arrowheads) and a round, enhanced lesion, suggesting a pseudoaneurysm (red arrowhead). (D) Proper hepatic artery angiogram.
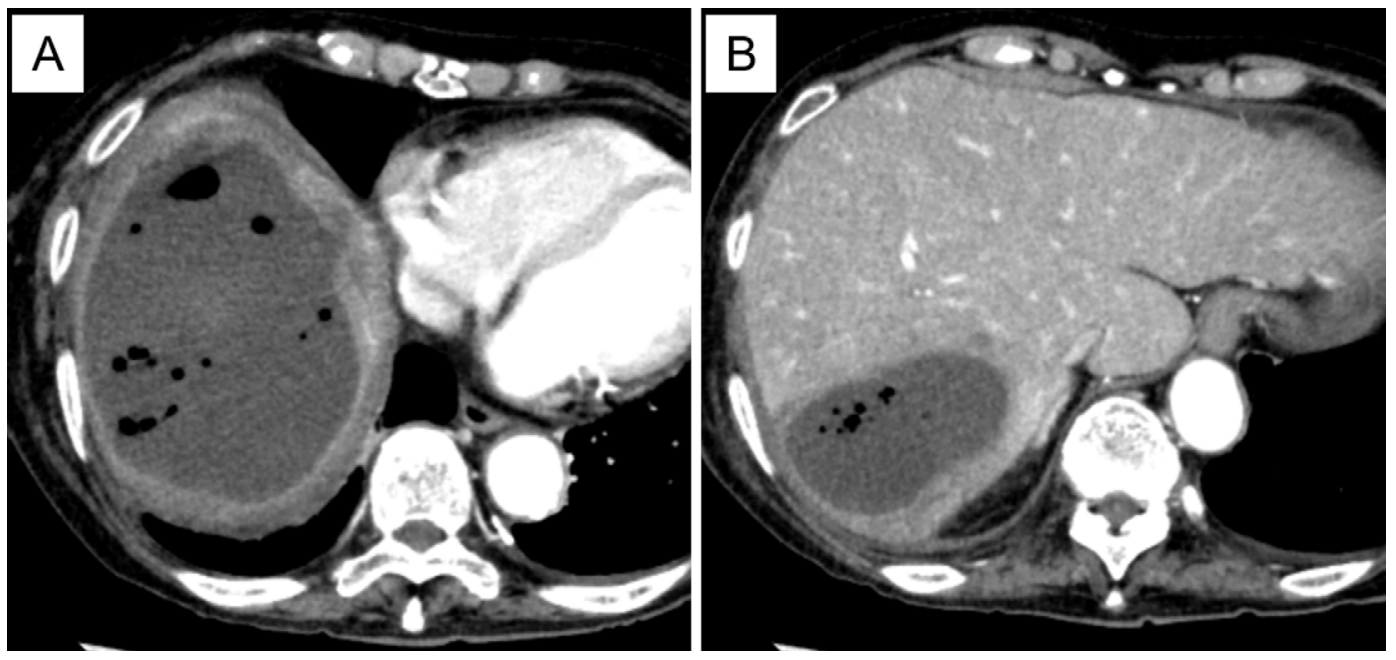

Figure 6. Abdominal CT images at the removal of the drainage catheter. The abscess cavity is filled with a substance containing small air bubbles. The gas that had formed the air-fluid level is no longer present. (A) Axial image, cephalic level. (B) Axial image, caudal level.

days. An early diagnostic clue may be Gram staining. $K$. pneumoniae and E. coli, the main causative bacteria of gasforming liver abscesses, are Gram-negative rods, whereas $C$. perfringens is a Gram-positive rod. In the present case,
Gram staining identified large, Gram-positive rods before the identification with pus culture, and given the gasformation, we suspected $C$. perfringens infection and were able to initiate the administration of appropriate antibiotics. 
The prediction of $C$. perfringens by Gram staining may be essential for the prompt treatment of $C$. perfringens infection.

The prognosis of patients with septicemia caused by $C$. perfringens is extremely poor, with a $70-100 \%$ mortality due to massive hemolysis caused by the alpha-toxin (3). The hemolysis is mainly caused by the disruption of erythrocyte membranes due to the phospholipase $\mathrm{C}$ activity of alphatoxin (6). A peripheral blood smear of the hemolysis shows spherocytes, ghost cells, and cyst cells $(12,13)$. Elevated levels of indirect bilirubin, $\mathrm{LDH}$, and potassium in the serum support the detection of hemolysis. In the present case, the bilirubin level was elevated, but it was predominantly direct bilirubin. Her peripheral blood smear did not show spherocytes or ghost cells, indicating no evidence of massive hemolysis.

Treatment of liver abscesses caused by $C$. perfringens is based on drainage and antibiotic administration. Percutaneous drainage should be promptly performed for the gasforming liver abscess. Liver abscesses caused by $C$. perfringens tend to rupture because of the vulnerable abscess cavity wall weakened by hepatocyte injury and increased internal pressure due to gas formation (15). Drainage or surgical removal of the abscess improves the prognosis (10). In general, percutaneous drainage is the first step in treating pyogenic liver abscesses, followed by more invasive surgical drainage or resection if this approach is not successful. $C$. perfringens pus can be a highly viscous fluid, so drainage may not be effective. In such cases, surgical treatment should be considered without delay. In the present case, the pus was too viscous to drain spontaneously through the placed drainage catheter. Since the patient was too old with too many complications to undergo hepatectomy, we continued flushing the catheter tube with saline. Although the abscess cavity did not disappear, the abscess fluid did solidify with long-term antibiotics administration.

C. perfringens is susceptible to penicillins, cephems, and carbapenems, but clindamycin and metronidazole have been reported to be superior at inhibiting the activity of toxins produced by $C$. perfringens at an early stage (16). Combining these drugs with penicillins, cephems, or carbapenems may be useful for preventing hemolysis from causing a critical condition $(10,16)$. In the present case, a carbapenem was started immediately after admission, and when Grampositive rods were detected in the pus, we suspected Clostridium infection and started administrating clindamycin/ metronidazole. Although the $C$. perfringens in this case may have been a strain with an alpha-toxin activity too low to cause hemolysis, the early initiation of appropriate antibiotics administration might have been the reason for the lack of massive hemolytic anemia.

In this case, bleeding into the abscess occurred due to the disruption of the right inferior phrenic artery during the clinical course. A markedly increased internal pressure due to gas formation and the spread of inflammation to the diaphragm may have caused the collapse of a branch of the right inferior phrenic artery. Bleeding in visceral arteries mainly occurs in splenic arteries, followed by hepatic arteries, and is rare in the inferior phrenic arteries $(17,18)$. The causes of bleeding in the inferior phrenic artery consist of pseudoaneurysms induced by mechanical injury including surgical procedures and vulnerable arteries induced by spreading inflammation due to pancreatitis or other entities (19). However, visceral arterial bleeding reports related to liver abscesses are limited to those involving hepatic arteries that often induce biliary bleeding. The spread of inflammation from the abscess generates pseudoaneurysm formation in hepatic arteries, resulting in bleeding (5). There have been no reports of inferior phrenic artery bleeding due to liver abscesses, suggesting that the spread of inflammation may be generally limited to the liver. In the present case, the abscess was located in the posterior segment close to the diaphragm, resulting in the spread of the inflammation to the diaphragm.

In conclusion, we encountered a rare case of gas-forming liver abscess caused by $C$. perfringens near the diaphragm, resulting in bleeding into the abscess cavity due to rupture of the inferior phrenic artery. Physicians should keep in mind that a liver abscess in the adjacent diaphragm can cause arterial bleeding.

Informed consent was obtained from the patient for publishing.

The authors state that they have no Conflict of Interest (COI).

\section{References}

1. Thng CB, Tan YP, Shelat VG. Gas-forming pyogenic liver abscess: a world review. Ann Hepatobiliary Pancreat Surg 22: 11-18, 2018.

2. Chen SC, Tsai SJ, Chen $\mathrm{CH}$, et al. Predictors of mortality in patients with pyogenic liver abscess. Neth J Med 66: 196-203, 2008.

3. Rogstad B, Ritland S, Lunde S, Hagen AG. Clostridium perfringens septicemia with massive hemolysis. Infection 21: 54-56, 1993.

4. Rajendran G, Bothma P, Brodbeck A. Intravascular haemolysis and septicaemia due to Clostridium perfringens liver abscess. Anaesth Intensive Care 38: 942-945, 2010.

5. Kang M, Bapuraj JR, Khandelwal N, Kochhar R, Kalra N, Verma GR. Liver abscess associated with hepatic artery pseudoaneurysm with arteriovenous fistula: imaging and interventional management. Acta Radiol 47: 162-166, 2006.

6. Sakurai J, Nagahama M, Oda M. Clostridium perfringens alphatoxin: characterization and mode of action. J Biochem 136: 569574, 2004.

7. Luo M, Yang XX, Tan B, et al. Distribution of common pathogens in patients with pyogenic liver abscess in China: a meta-analysis. Eur J Clin Microbiol Infect Dis 35: 1557-1565, 2016.

8. Rahimian J, Wilson T, Oram V, Holzman RS. Pyogenic liver abscess: recent trends in etiology and mortality. Clin Infect Dis 39: 1654-1659, 2004.

9. Rechner PM, Agger WA, Mruz K, Cogbill TH. Clinical features of clostridial bacteremia: a review from a rural area. Clin Infect Dis 33: 349-353, 2001.

10. Simon TG, Bradley J, Jones A, Carino G. Massive intravascular hemolysis from Clostridium perfringens septicemia: a review. J In- 
tensive Care Med 29: 327-333, 2014.

11. Awad MM, Bryant AE, Stevens DL, Rood JI. Virulence studies on chromosomal alpha-toxin and theta-toxin mutants constructed by allelic exchange provide genetic evidence for the essential role of alpha-toxin in Clostridium perfringens-mediated gas gangrene. Mol Microbiol 15: 191-202, 1995.

12. Hashiba M, Tomino A, Takenaka N, et al. Clostridium perfringens infection in a febrile patient with severe hemolytic anemia. Am J Case Rep 17: 219-223, 2016.

13. van Bunderen CC, Bomers MK, Wesdorp E, Peerbooms $P$, Veenstra J. Clostridium perfringens septicaemia with massive intravascular haemolysis: a case report and review of the literature. Neth J Med 68: 343-346, 2010.

14. Kwon YK, Cheema FA, Maneckshana BT, Rochon C, Sheiner PA. Clostridium paraputrificum septicemia and liver abscess. World J Hepatol 10: 388-395, 2018.

15. Yang CC, Chen CY, Lin XZ, Chang TT, Shin JS, Lin CY. Pyogenic liver abscess in Taiwan: emphasis on gas-forming liver ab- scess in diabetics. Am J Gastroenterol 88: 1911-1915, 1993.

16. Stevens DL, Maier KA, Mitten JE. Effect of antibiotics on toxin production and viability of Clostridium perfringens. Antimicrob Agents Chemother 31: 213-218, 1987.

17. Arneson MA, Smith RS. Ruptured hepatic artery aneurysm: case report and review of literature. Ann Vasc Surg 19: 540-545, 2005.

18. Baker KS, Tisnado J, Cho SR, Beachley MC. Splanchnic artery aneurysms and pseudoaneurysms: transcatheter embolization. Radiology 163: 135-139, 1987.

19. Kearney PA, Rouhana SW, Burney RE. Blunt rupture of the diaphragm: mechanism, diagnosis, and treatment. Ann Emerg Med 18: 1326-1330, 1989.

The Internal Medicine is an Open Access journal distributed under the Creative Commons Attribution-NonCommercial-NoDerivatives 4.0 International License. To view the details of this license, please visit (https://creativecommons.org/licenses/ by-nc-nd/4.0/).

(C) 2021 The Japanese Society of Internal Medicine

Intern Med 60: 3913-3919, 2021 